\title{
Tuberculosis related stigma
}

\author{
Pinar AY (D), Seyhan HIDIROGLU (D) \\ Department of Public Health, School of Medicine, Marmara University, Maltepe, Istanbul, Turkey.
}

Corresponding Author: Pinar AY

E-mail: npay@marmara.edu.tr

\begin{abstract}
While tuberculosis is a preventable and curable disease, it still remains to be a major cause of morbidity and mortality across the world. Research reveals that stigma impacts tuberculosis control negatively through delaying diagnosis and impairing treatment compliance. It also causes enormous suffering since, it results with isolation of patients and their families from social life. In this review; the magnitude, origins, correlates and consequences of tuberculosis related stigma are discussed and some examples of public health interventions to combat stigma are presented.

Keywords: Tuberculosis, Stigma, Public Health implications
\end{abstract}

\section{INTRODUCTION}

While tuberculosis (TB) is a preventable and a curable disease, it still continues to have a high burden particularly in low and middle income countries. World Health Organization (WHO) estimated that in 2016, there were more than 10 million new TB cases globally. In the same year, TB lead to 1.3 million deaths and was among the top ten causes of mortality worldwide [1]. The most effective public health approach to prevent and control $\mathrm{TB}$ is the early detection and effective treatment of the patients. However, TB related stigma poses an important barrier to the prevention and control efforts through delaying diagnosis and impairing treatment adherence.

Patients suffering from TB might be reluctant to seek care and apply for diagnostic services with the fear of getting stigmatized. Stigma acts as a barrier to early diagnosis and patients remain infectious within the community for longer periods of time transmitting the disease. Stigma also impairs treatment adherence. Non-compliance to therapy contributes both to the increased risk of transmission and the development of multidrug resistant $\mathrm{TB}$, of which treatment options are limited, toxic and very expensive [2-9]. Furthermore, due to the stigma attached to TB, some of the patients conceal their diagnosis from their family members and/or co-workers which hinders contact-tracing and detection of new cases [10]. Therefore, in order to control TB effectively, it is crucial to fully explore, understand and address disease related stigma [11].
Stigma is initially defined by Goffman as the situation of the individual who is disqualified from full social acceptance [12]. Link and Phelan broadened this definition and conceptualized it as a social process that exists when elements of labelling, stereotyping, separation, status loss, and discrimination occur in a power situation that allows them to unfold $[13,14]$. There are mainly two types of stigma defined; enacted and perceived (internalized) stigma. Enacted stigma refers to the discrimination or devaluation experienced by the TB patients on the grounds of their perceived unacceptability or inferiority. Felt (perceived or internalized) stigma is a sense of shame or withdrawal of TB patients which results from the fear of enacted stigma $[15,16]$. Stigma is not only experienced by the patients. Patients' connections, for example family members also suffer from TB related stigma. Goffman describes courtesy stigma as society degrading or losing respect for a person because the person associates with someone who is stigmatized [12].

\section{Magnitude of TB stigma}

Stigma is a social process that has multi dimensions and unclear borders, so it cannot be quantified explicitly [17]. There is not a standard method of measurement; while some studies use validated stigma scales others rely only on a few questions. Due to these methodological differences, it is neither possible to give a global figure nor to make comparisons across studies

How to cite this article: Ay P, Hidiroglu S. Tuberculosis related stigma. Marmara Med J 2021;34 (Special Issue); doi: 10.5472/marumj.854373 
concerning the magnitude of stigma. Still, research reveals that although there are wide variations, TB related stigma is present in different cultures across the world [18-25].

Studies mostly evaluate stigma among TB patients who apply for care. A study carried out among newly diagnosed TB cases in seven countries of the WHO Eastern Mediterranean Region showed that 85 to $95 \%$ of the patients felt ashamed that they had developed TB and tried to conceal the disease [20]. A survey among patients attending to Directly Observed Treatment Short-Course (DOTS) clinics in a municipality in Nepal revealed that two thirds of the patients had experienced some kind of TB related stigma. Among the participants, $60 \%$ desired not to disclose their disease to other people and $43 \%$ thought that others had avoided them because of their disease. More than one third of the patients indicated that they were asked to stay away from work [18]. Another study carried out among TB patients in a district in India revealed that approximately half of the participants had experienced some form of TB related stigma [22].

Studies from Turkey, which is a low incidence country, also reveal that stigma is experienced among TB patients. A study evaluating internalized stigma of TB patients who had registered to a TB dispensary in a province located in western Turkey determined that more than one third of the patients did not disclose their diagnosis to other people [23]. Another study evaluating stigma among patients attending a TB clinic in a province of Turkey also showed that half of the participants did not disclose their disease [24].

Although, relatively few in number, there are also studies that evaluate the extent of stigma community members attached to TB patients. A community based survey which was carried out in 30 districts in India revealed that the proportion of respondents in the general population having stigmatizing and discriminating attitudes to TB sufferers were $73 \%$ and $98 \%$, respectively [26]. A study analyzing surveys from 13 different countries that evaluated TB courtesy stigma revealed that about one fourth of the participants would not disclose the TB status of their family members to others [21].

Studies show that health care workers might also have stigmatizing and discriminating attitudes towards TB patients $[19,27,28]$. A study carried out among resident doctors in India revealed that half of the respondents experienced fear, lack of compassion and tendency to avoid TB patients. Unfavorable attitudes were more prevalent among doctors who knew a colleague with $\mathrm{TB}$ compared to the ones who did not. The authors suggested that the doctors who had a colleague with TB perceived the occupational transmission more real, which showed the importance of improving the occupational safety measures [28]. TB patients also confirm that they experience stigmatizing attitudes of health care workers. In a study in Nepal, some patients stated that they felt to be discriminated by health care workers particularly at the time of diagnosis [27].

As discussed above, most of the stigma research evaluates TB patients who apply to health care centers. The magnitude, determinants and the impact of stigma are not explored explicitly among the patients who do not seek care and also the community members [29]. In order to build effective interventions to combat stigma Macintyre et al., suggest the need of widening the TB stigma research to the general public, policy makers, media actors and also to health care workers [29].

\section{Origins and correlates of TB stigma}

Research show that the perceived risk of transmission of TB to family members, friends and to the other loved ones is the main determinant leading to stigmatization [3,27]. Some patients are deeply concerned about transmitting the disease particularly to their children [27].

Other bases of stigma are mostly related to the socioeconomic determinants of TB. In some cultures, TB in a way serves as a marker for poverty, malnutrition and being foreign-borne which are also stigmatized conditions $[3,27,30,31]$. In Nepal, TB is related to being poor and of low caste [27]. In South Africa, TB is believed to have resulted from dirt, poor sanitation and squalor [31]. In some cultures having a family member with TB is linked to not being able to meet the economic requirements of the family. A study conducted among Haitians showed that having a family member diagnosed with TB indicated one's inadequacy to fulfill the needs of the family for food or sufficient living space which had led to shame and guilt [30].

Some communities view TB as a "dirty disease" and believe that it has resulted from immoral practices or bad behavior [32]. A study in Nepal revealed that TB was linked with "immoral behavior", which meant smoking, drinking alcohol and visiting prostitutes [27]. A qualitative study in Tanzania showed that TB was believed to be sexually transmitted and linked to promiscuity for women [33]. Such perceptions result with the feelings of shame and embarrassment both among the patients and the family members. In relation to this, in some cultures the disease is perceived as a result of a "divine curse" due to immoral practices [27, 32].

Patients might face a greater level of stigma if they have resistant forms of TB $[29,34]$. Macintry et al., indicate that if a patient has multi-drug resistant TB (MDR-TB) or extensively drug-resistant tuberculosis (XDR-TB), then the perceived dangerousness increases and contributes to the stigma experienced. Also, since these forms of TB might originate from non-adherence to treatment, patients might be accused of contributing to the development of drug resistance. Drug adverse effects and high economic impact might also increase the vulnerability to stigma [29].

Comorbidity with HIV contributes to the TB related stigma [3, $21,30,31,35]$. Patients fear that if they are identified with TB, community members would assume that they also have HIV [30]. A qualitative study carried out in South Africa revealed that TB led to some physical symptoms as weight loss, which also caused HIV to become more noticeable. The authors indicate that co-infection with $\mathrm{HIV}$ and $\mathrm{TB}$ created an overlapping double stigma which consequently facilitated new forms of TB stigma and intensified the existing stigma against HIV [36]. 
The impact of TB knowledge regarding symptoms, transmission and curability on stigma does not show a consistent relationship across different communities. While, some studies indicate that better knowledge on TB is associated with a lower level of stigma, others do not show such an association [21, 25, 26, 35]. So, there is also a need to clearly understand whether providing more information about TB would reduce stigma [3].

\section{Consequences of TB stigma}

Having TB is mainly linked to the feeling of shame and guilt which consequently leads TB patients to isolate themselves from social life $[20,32,33,37,38]$. Isolation from social life occurs by two means; while some patients withdraw themselves from interaction, some others are shunned, not being welcomed in the community [32]. Isolation might last throughout the whole treatment period, although, patients are told that they are not infective after the first couple of weeks of the treatment initiation [27]. So, eventually both the patient and the patient's family get detached from the community. If there is stigmatization also within the family, the family members become isolated from each other [32]. The patient might be obliged to have separate utensils and sleep in a separate bedroom [6]. Females having TB might have lower chances of getting married or if married can be rejected by their partners and may be at an increased risk for divorce $[19,20,33,38]$. In a study which was carried out in seven countries of the WHO Eastern Mediterranean Region, $96 \%$ of females and $90 \%$ of males believed that the chances of a girl diagnosed with TB getting married were low [20].

Self-stigmatization, fear of discrimination and also trying to protect the family from TB related stigma mostly result with the concealment of the disease. Patients do not disclose their disease to the community; neighbors, relatives, co-workers and in some instances even to their own family members $[4,6,27$, $30,38-41]$. Keeping TB diagnosis as a secret from the family and friends increases the social isolation even more and prevents the patients to get the social support they need [41]. Stigma also increases the psychological burden on patients. A study carried out among TB patients in a rural area in China determined that experienced stigma was independently associated with psychological distress [42].

Tuberculosis related stigma can also impact economic opportunities [3, 37]. A study carried out in Sri Lanka showed the huge impact of TB diagnosis on economic status. The study pointed out that among the participants $62.8 \%$ changed their job due to TB and $52.3 \%$ had changes to their income level [40].

Tuberculosis stigma has a negative impact on initiating a diagnostic process and also treatment adherence. A multicountry study carried out in seven countries of the WHO Eastern Mediterranean Region indicated that stigma was one of the main determinants of delay to access care [20]. A qualitative study in Eritrea showed that fear of being recognized in the community prevented TB patients to attend health care services and also take their medications [6]. A study in Tanzania showed that stigma resulted with a delay in health care seeking behavior with the fear of getting diagnosed with TB [33]. Even health care workers might be reluctant to use diagnostic services because of the TB related stigma. A study in South Africa evaluated TB related stigma of health care workers working in six hospitals and their willingness to use occupational health services for TB screening, treatment and prophylaxis. The study revealed that perceived TB stigma among health care workers decreased the willingness to use occupational health services particularly for TB screening [43].

Stigma might also have an impact in low TB incidence countries. A contact investigation in a workplace in Italy determined three additional cases and 33 latent infections that had occurred among the employees during the previous five years. The source case claimed to be unemployed so contact-tracing had not been carried out at the time of the diagnosis in the workplace. It was revealed that the source case had a temporary work contract and feared that her contract might not be renewed if she had disclosed her disease. The authors indicated that job insecurity as the predominant factor leading to this outbreak [10].

It is also important to note that the impact of stigma is not the same on every group. Courtwright and Turner indicated in their study that the impact of TB stigma was more prominent on women and socioeconomically disadvantaged group [3]. A study in Tanzania showed that TB stigma operated differently among men and women. While, for women TB related stigma leads to problems in partner relationships, for men it threatens their survival through affecting their daily activities [33]. So, TB stigma might deepen the already existing disparities in health resulting with a higher burden on the most deprived groups [3].

\section{Public Health interventions to reduce TB stigma}

Although, TB stigma is prevalent across the world, studies evaluating the impact of stigma reduction strategies are limited in number. Sommerland et al., reviewed interventions to reduce TB stigma and identified only seven studies published between 1950 and 2015. These studies implemented different methods to combat stigma; while some used knowledge shaping or attitude changing approaches, others relied on patient-support activities. The authors indicated that some of these studies showed promising results, but still most of them were lacking reliable information due to deficiencies of using rigorous evaluation methods. Also, the interventions showing a reduction in stigma levels were not replicated. The authors highlighted the need to conduct studies which evaluated interventions objectively using evidence based tools and also to replicate the successful interventions in different parts of the world [43].

Among the stigma reduction strategies, "TB clubs" is an innovative approach deserving special attention. The effectiveness of TB clubs in improving adherence to treatment and decreasing stigma were studied initially in a rural region in Ethiopia. TB clubs were formed from a group of patients residing in the same region. The clubs met on weekly bases to share information and provide mutual support. Monthly meetings were also held with health care workers. TB club members also interacted with leaders and members of the community to exchange information and disseminate educational materials. The authors documented that the intervention was successful in reducing $\mathrm{TB}$ related stigma and improving treatment adherence. 
While patients were not disclosing their disease previously, with the introduction of the intervention they started talking about their disease in public gatherings [5]. A similar intervention in rural Nicaragua also yielded positive results for reducing internalized stigma of TB patients. In this study, the authors utilized two approaches to combat stigma; TB clubs and patient centered home visits. TB clubs were designed as self-help groups to empower patients. Patient centered home visits were performed with the aim of understanding the patients' lives and their socio-cultural context in order to plan activities to support them. The authors documented that after two months, internalized stigma scores in the intervention group decreased significantly while a difference was not observed in the control group [16].

Although, these studies show promising results in empowering patients to resist stigma, Courtwright and Turner underline the need to study the impact of TB clubs in other regions of the world [3]. It is important to remember that a successful intervention in a community might not necessarily generate similar results in another region because different meanings are attached to stigma based on the social context for different ethnic and cultural groups [44]. Even within the same cultural group, TB related stigma might be perceived and experienced differently among men and women and across different age groups. So, interventions addressing stigma should take into account gender, age, social context and their interaction with stigma $[33,44]$. It is also essential to explore how these strategies impact the end results. We need to understand whether the reduction of stigma results with a rapid access to care and treatment, whether it increases treatment adherence and decreases morbidity and mortality [3].

Daftary et al., indicated that to confront TB related stigma we need to understand how the HIV community confronted HIV related stigma and take lessons from that experience. The authors argued that consciousness raising as a form of activism is necessary to mitigate $\mathrm{TB}$ related stigma. In this context, people affected by TB are expected to come together and form a movement of consciousness raising. This collective movement, through sharing their experiences can begin organizing forces of change that will reduce TB related stigma [45].

\section{Conclusion}

Although, TB related stigma is a global problem, studies evaluating interventions to reduce it are insufficient both in number and quality. There is a need to form innovative methods to combat TB related stigma based on the cultural context of the community and evaluate them using evidence based methods in different parts of the world.

Funding: The authors received no financial support for this review.

Conflict of Interest: The authors declare that they have no conflict of interest.

\section{REFERENCES}

[1] Organization WHO. Global Tuberculosis Report 2017. 2017.

[2] Chakrabartty A, Basu P, Ali KM, Sarkar AK, Ghosh D. Tuberculosis related stigma and its effect on the delay for sputum examination under the Revised National Tuberculosis Control Program in India. Indian J Tuberc 2018;65:145-51.

[3] Courtwright A, Turner AN. Tuberculosis and stigmatization: pathways and interventions. Public Health Rep (Washington, DC : 1974). 2010;125 Suppl 4:34-42. doi: 10.1177/003.335.49101250S407.

[4] de Vries SG, Cremers AL, Heuvelings CC, et al. Barriers and facilitators to the uptake of tuberculosis diagnostic and treatment services by hard-to-reach populations in countries of low and medium tuberculosis incidence: a systematic review of qualitative literature. Lancet Infect Dis 2017;17:e128-e43. doi: 10.1016/S1473-3099(16)30531-X.

[5] Demissie M, Getahun H, Lindtjorn B. Community tuberculosis care through "TB clubs" in rural North Ethiopia. Soc Sci Med 2003;56:2009-18. doi: 10.1016/s0277-9536(02)00182-x.

[6] Gebreweld FH, Kifle MM, Gebremicheal FE, et al. Factors influencing adherence to tuberculosis treatment in Asmara, Eritrea: a qualitative study. J Health Popul Nutr 2018;37(1):1. doi: 10.1186/s41043.017.0132-y.

[7] Sabawoon W, Sato H, Kobayashi Y. Delay in the treatment of pulmonary tuberculosis: a report from Afghanistan. Environ Health Prev Med 2012;17:53-61. doi: 10.1007/ s12199.011.0219-9.

[8] Saqib SE, Ahmad MM, Amezcua-Prieto C, Virginia MR. Treatment delay among pulmonary tuberculosis patients within the Pakistan National Tuberculosis Control Program. Am J Trop Med Hyg 2018;99:143-9. doi: 10.4269/ ajtmh.18-0001.

[9] Yan S, Zhang S, Tong Y, Yin X, Lu Z, Gong Y. Nonadherence to antituberculosis medications: The impact of stigma and depressive symptoms. Am J Trop Med Hygi 2018;98:262-5. doi: 10.4269/ajtmh.17-0383.

[10] Faccini M, Cantoni S, Ciconali G, et al. Tuberculosis-related stigma leading to an incomplete contact investigation in a low-incidence country. Epidem Infect 2015;143:2841-8. doi: 10.1017/S095.026.881400394X.

[11] Jaramillo E, Sahu S, Van Weezenbeek C. Ending TBrelated stigma and discrimination. Int J Tuberc Lung Dis 2017;21(11):2-3. doi: 10.5588/ijtld.17.0229.

[12] Goffman E. Stigma: Notes on the management of spoiled identity. NJ:Prentice Hall Englewood Cliffs , 1963.

[13] Link BG, Phelan PJ. Conceptualizing stigma. Annu Rev Sociol 2001;27:363-85.

[14] Link BG, Phelan JC. Stigma and its public health implications. Lancet 2006;367(9509):528-9.

[15] Scambler G HA. Being epileptic: coming to terms with stigma. Sociology of Health and Illness 1986;8:26-43.

[16] Macq J, Solis A, Martinez G, Martiny P. Tackling tuberculosis patients' internalized social stigma through patient centred 
care: an intervention study in rural Nicaragua. BMC Public Health 2008;8:154.

[17] Mitchell EMH, Daftary A. TB stigma: clearing the fog. Int J Tuberc Lung Dis 2017;21:1. doi: 10.5588/ijtld.17.0651

[18] Aryal S, Badhu A, Pandey S, Bhandari A, et al. Stigma related to tuberculosis among patients attending DOTS clinics of Dharan municipality. Kathmandu Univ Med J 2012;10:48-52. doi: 10.3126/kumj.v10i1.6914.

[19] Burtscher D, Van den Bergh R, Toktosunov U, Angmo N, Samieva N, Rocillo Arechaga EP. "My Favourite Day Is Sunday": Community perceptions of (Drug-Resistant) tuberculosis and ambulatory tuberculosis care in Kara Suu District, Osh Province, Kyrgyzstan. PloS One. 2016;11:e0152283. doi: 10.1371/journal.pone.0152283.

[20] EM/TDR/009/E/10.06/1000 WHOROftEM. Diagnostic and treatment delay in tuberculosis. Cairo, Egypt: WHO EMRO; 2006.

[21] Rood EJJ, Mergenthaler C, Bakker MI, Redwood L, Mitchell EMH. Using 15 DHS surveys to study epidemiological correlates of TB courtesy stigma and health-seeking behaviour. Int J Tuberc Lung Dis 2017;21:60-8. doi: 10.5588/ijtld.16.0909.

[22] Shivapujimath R, Rao AP, Nilima AR, Shilpa DM. A cross-sectional study ro assess the stigma associated with tuberculosis among tuberculosis patients in Udupi district in Karnataka. Indian Journal of Tuberculosis 2017;64:323-6. doi. org/10.1016/j.ijtb.2016.10.002

[23] Şimşek H ÖD, Çetinkaya AÇ. Evaluation internalized stigma of tuberculosis patients. Int j Nursing Res 2016;7:156-73.

[24] Yiğit Açıkel G ÇPS. Akciğer tüberkülozlu hastalarda damgalamanın değerlendirilmesi. FN Hem Derg 2015;23:13645.

[25] Yin X, Yan S, Tong Y, Peng X, Yang T, Lu Z, et al. Status of tuberculosis-related stigma and associated factors: a crosssectional study in central China. Trop Med Int Health 2018;23:199-205. doi: 10.1111/tmi.13017.

[26] Sagili KD, Satyanarayana S, Chadha SS. Is knowledge regarding tuberculosis associated with stigmatising and discriminating attitudes of general population towards tuberculosis patients? Findings from a community based survey in 30 districts of India. PloS one 2016;11(2):e0147274. doi: 10.1371/journal. pone. 0147274 .

[27] Baral SC, Karki DK, Newell JN. Causes of stigma and discrimination associated with tuberculosis in Nepal: a qualitative study. BMC Public Health 2007;7:211. doi: 10.1186/1471-2458-7-211.

[28] Pardeshi GS, Kadam D, Chandanwale A, Bollinger R, Deluca A. Resident doctors' attitudes toward tuberculosis patients. Indian J Tuberc 2017;64(2):89-92. doi: 10.1016/j. ijtb.2016.11.001

[29] Macintyre K, Bakker MI, Bergson S, et al. Defining the research agenda to measure and reduce tuberculosis stigmas. The international journal of tuberculosis and lung disease. Int J Tuberc Lung Dis 2017;21(11):87-96. doi: 10.5588/ ijtld.17.0151.
[30] Coreil J, Mayard G, Simpson KM, Lauzardo M, Zhu Y, Weiss M. Structural forces and the production of TB-related stigma among Haitians in two contexts. Soc Sci Med 2010;71:140917. doi: 10.1016/j.socscimed.2010.07.017.

[31] Murray EJ, Bond VA, Marais BJ, Godfrey-Faussett P, Ayles HM, Beyers N. High levels of vulnerability and anticipated stigma reduce the impetus for tuberculosis diagnosis in Cape Town, South Africa. Health Policy Plan 2013;28:410-8. doi: 10.1093/heapol/czs072.

[32] Juniarti N, Evans D. A qualitative review: the stigma of tuberculosis. J Clin Nurs 2011;20:1961-70. doi: 10.1111/j.13652702.2010.03516.x.

[33] Miller C, Huston J, Samu L, Mfinanga S, Hopewell P, Fair E. 'It makes the patient's spirit weaker': tuberculosis stigma and gender interaction in Dar es Salaam, Tanzania. Int J Tuberc Lung Dis 2017;21:42-8. doi: 10.5588/ijtld.16.0914.

[34] Skinner D, Claassens M. It's complicated: why do tuberculosis patients not initiate or stay adherent to treatment? A qualitative study from South Africa. BMC Infect Dis 2016;16:712. doi. org/10.1186/s12879.016.2054-5

[35] Kipp AM, Pungrassami P, Nilmanat K, et al. Sociodemographic and AIDS-related factors associated with tuberculosis stigma in southern Thailand: a quantitative, crosssectional study of stigma among patients with TB and healthy community members. BMC Public Health 2011;11:675. doi. org/10.1186/1471-2458-11-675

[36] Daftary A. HIV and tuberculosis: the construction and management of double stigma. Soc Sci Med (1982). 2012;74:1512-9. doi: 10.1016/j.socscimed.2012.01.027.

[37] Gerrish K, Naisby A, Ismail M. The meaning and consequences of tuberculosis among Somali people in the United Kingdom. J Adv Nurs 2012;68:2654-63. doi: 10.1111/j.13652648.2012.05964.x.

[38] Munro SA, Lewin SA, Smith HJ, Engel ME, Fretheim A, Volmink J. Patient adherence to tuberculosis treatment: a systematic review of qualitative research. PLoS Med 2007;4:e238. doi: 10.1371/journal.pmed.0040238.

[39] Gerrish K, Naisby A, Ismail M. Experiences of the diagnosis and management of tuberculosis: a focused ethnography of Somali patients and healthcare professionals in the UK. J Adv Nurs 2013;69:2285-94. doi: 10.1111/jan.12112.

[40] Senanayake MGB, Wickramasinghe SI, Samaraweera S, De Silva P, Edirippulige S. Examining the social status, risk factors and lifestyle changes of tuberculosis patients in Sri Lanka during the treatment period: a cross-sectional study. Multidiscip Respir Med 2018;13:9. doi: 10.1186/ s40248.018.0121-z.

[41] Zuniga JA, Munoz SE, Johnson MZ, Garcia A. Tuberculosis treatment for Mexican Americans living on the U.S.-Mexico border. J Nurs Scholarsh 2014;46:253-62. doi: 10.1111/ jnu.12071.

[42] Xu M, Markstrom U, Lyu J, Xu L. Survey on Tuberculosis patients in rural areas in China: tracing the role of stigma in psychological distress. Int J Enviro Res Public Health 2017;14:1171. doi:10.3390/ijerph14101171 
[43] Sommerland N, Wouters E, Mitchell EMH, et al. Evidencebased interventions to reduce tuberculosis stigma: a systematic review. Int J Tuberc Lung Dis 2017;21(11):81-6. doi: 10.5588/ ijtld.16.0788.

[44] Chang SH, Cataldo JK. A systematic review of global cultural variations in knowledge, attitudes and health responses to tuberculosis stigma. lung disease. Int J Tuberc Lung Dis 2014;18:168-73, i-iv. doi: 10.5588/ijtld.13.0181.

[45] Daftary A, Frick M, Venkatesan N, Pai M. Fighting TB stigma: we need to apply lessons learnt from HIV activism. BMJ Glob Health 2017;2:e000515. doi: 10.1136/bmjgh-2017-000515. 